Pacific Journal of Mathematics

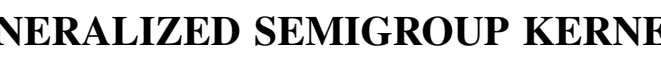




\title{
GENERALIZED SEMIGROUP KERNELS
}

\author{
RONALD FULP
}

This paper is concerned with the problem of generalizing the notion of a kernel of a semigroup. Various kernels are introduced and their mutual relationships are investigated. Conditions are found on a semigroup which are necessary and sufficient in order that certain of its kernels be trivial.

The "generalized" kernels we introduce here have properties which are reminiscent of the notion of a radical. Our results, however, are quite different from certain of the investigations along these lines (see, for example, [3] and [13]). Our work is more closely related to that of Schwartz [10], [11], and [12]. We refer to [2] for definitions not explicitly given.

1. Mutually annihilating sums and kernels. The following definition seems to be due to Ljapin [6]. If $S$ is a semigroup, then $S$ is said to be a mutually annihilating sum of semigroups $\left\{S_{\lambda}\right\}_{\lambda \in_{A}}$ if and only if $S$ is (isomorphic to) a semigroup with zero such that if 0 is the zero of $S$, then

(i) for $\lambda$ in $\Lambda, S_{\lambda}$ is a subsemigroup of $S$ with contains 0 ,

(ii) each member of $S$ is in $S_{\lambda}$ for some $\lambda$ in $A$, and

(iii) for $\lambda$ and $\gamma$ in $\Lambda, \lambda \neq \gamma, S_{\lambda} \cap S_{\lambda}=\{0\}=S_{\lambda} S_{\gamma}$.

We shall be concerned with semigroups $S$ which are mutually annihilating sums of semigroups each of which has some one fixed semigroup property $P$ (to say that $P$ is a semigroup property means that $P$ is a property such that if one of two isomorphic semigroups has property $P$, then so does the other). There is a rather obvious connection between mutually annihilating sums and subdirect sums which we make explicit in the lemma below.

We use the concept of a subdirect sum as in the theory of rings, i.e., to say that $S$ is a subdirect sum of semigroups $\left\{T_{\mu}\right\}_{\mu \in \Omega}$ means that $S$ is (isomorphic to) a subsemigroup of the direct product $\Pi_{\mu \in \Omega} T_{\mu^{\prime}}$ such that if for some $\nu \in \Omega, \pi_{\nu}$ is the projection of $\Pi_{l^{\prime \prime} \in \Omega} T_{\|}$onto $T_{\nu}$, then the homomorphism $\pi_{\nu} \mid S$ is onto $T_{\nu}$. The following lemma is not difficult to prove.

LEMmA 1.1 If $S$ is a semigroup with zero, then $S$ is a mutually annihilating sum of semigroups each having property $P$ if and only if there is a collection $\left\{T_{\mu}\right\}_{\mu \in \Omega}$ of semigroups such that

(1) for each $\mu \in \Omega, T_{\mu}$ is a semigroup with zero which has property $P$, and 
(2) $S$ is a subdirect sum of the collection $\left\{T_{\mu}\right\}_{\mu \in \Omega}$ such that each member of $S$, when viewed as a member of $\Pi_{\mu \in \Omega} T_{\mu}$, has at most one nonzero component.

Let $K_{P}$ denote the set $\{I \mid I$ is an ideal and $S / I$ has property $P\}$.

THEOREM 1.2. Suppose $P$ is a semigroup property, $S$ is a semigroup, and $J$ is an ideal of $S$. Then $S / J$ is a mutually annihilating sum of semigroups each having property $P$ if and only if there is a subset $K$ of $K_{P}$ such that (i) $J$ is the K-kernel of $S$, and (ii) if $I$ and $I^{\prime}$ are distinct members of $K$, then $S=I \cup I^{\prime}$.

Proof. Assume $K$ is a subset of $K_{P}$ such that (i) and (ii) of the theorem are true. It is clear from (i) that $S / J$ is, in a natural way, a subdirect sum of the collection $\{S / I \mid I \in K\}$. Property (ii) implies that each member of the subdirect sum has no more than one nonzero component. It then follows from (i) and the lemma that $S / J$ is a mutually annihilating sum of semigroups each having property $P$.

Now assume $J$ is an ideal of $S$ and $S / J$ is a mutually annihilating

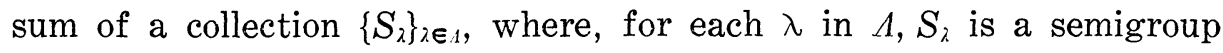
having property $P$. Let $\varphi$ denote the natural homomorphism from $S$ onto $S / J$. For each $\lambda \in \Lambda$, let $I_{\lambda}$ denote the set of all $x$ in $S$ such that either $\varphi(x)$ is zero or $\varphi(x)$ is not in $S$. If $K=\left\{I_{\lambda} \mid \lambda \in \Lambda\right\}$, then $K$ satisfies (i) and (ii) of the theorem.

REMARK 1.3. In case $S$ has a zero and $J$ is zero, the theorem asserts that $S$ is a mutually annihilating sum of semigroups each of which has property $P$ if and only if there is a subset $K$ of $K_{P}$ such that (i) $\cap K=0$ and (ii) if $I$ and $I^{\prime}$ are in $K, I \neq I^{\prime}$, then $S=I \cup I^{\prime}$.

For each semigroup $S$, let $\mathscr{C}=\mathscr{C}_{S}$ denote the set of all maximal ideals of $S$. The following corollaries are immediate applications of Remark 1.3.

Corollary 1.4. Assume $S$ is a semigroup with zero. Then $\cap \mathscr{C}=0$ if and only if $S$ is a mutually annihilating sum of semigroups each of which either is a null semigroup of order two or is a 0-simple semigroup.

COROLlaRY 1.5. If $S$ is a semigroup and $J$ is the Mll-kernel of $S$, then the $\mathscr{C l}$-kernel of $S / J$ is zero.

The $\mathscr{C}$-kernel of a semigroup determines, to some extent, which maximal ideals are prime (an ideal $J$ of a semigroup $S$ is said to be prime if and only if either $J=S$ or the complement of $J$ is a subsemigroup of $S$ ). 
THEOREM 1.6. Suppose $S$ is a semigroup which has a maximal ideal. If $J$ denotes the $\mathscr{C}$-kernel of $S$, then each maximal ideal of $S$ is prime if and only if there is a collection $\left\{S_{\alpha}\right\}_{\alpha \in_{\Lambda}}$ of simple subsemigroups of $S$ such that

(1) $S=J \cup \bigcup_{\alpha \in \Lambda} S_{\alpha}$,

(2) for $\alpha \in \Lambda, \beta \in \Lambda, \alpha \neq \beta, S_{\alpha} \cap S_{\beta}$ is void and $S_{\alpha} S_{\beta} \subseteq J$, and

(3) for each $\alpha \in \Lambda, J \cap S_{\alpha}$ is void.

Proof. First assume that each maximal ideal of $S$ is prime and that $J$ is the $\mathscr{C}$-kernel of $S$. From previous arguments, it is known that $S / J$ is isomorphic to a mutually annihilating sum of semigroups each of which is isomorphic to $S / M$ for some $M \in \mathscr{l l}$. Since $S / M$ is a simple semigroup with zero for each $M \in \mathbb{l}$ (recall that $M$ is prime), it follows that there is a collection $\left\{S_{\alpha}\right\}_{a \in \Lambda}$ of simple semigroups such that $S / J$ is a mutually annihilating sum of $\left\{S_{\alpha}^{0} \mid \alpha \in \Lambda\right\}$. For each $\alpha \in \Lambda$, we identify $S_{\alpha}$ with the subsemigroup $T_{\alpha}$ of $S$ such that

$$
\left(T_{\alpha} \cup J\right) / J=S_{\alpha}
$$

and $T_{\alpha} \cap J$ is void. Then the collection $\left\{S_{\alpha}\right\}_{\alpha \in A}$ satisfies (1), (2), and (3) of the theorem.

Assume, on the other hand, that $\left\{S_{\alpha}\right\}_{\alpha \in A}$ is a collection of simple subsemigroups of $S$ such that (1), (2), and (3) hold where $J$ denotes the $\mathscr{C}$-kernel of $S$. Then each maximal ideal of $S$ is of the form

$$
J \cup \bigcup_{\alpha \in \Lambda \backslash \beta\}} S_{\alpha}
$$

for some $\beta \in \Lambda$. Thus each maximal ideal of $S$ is prime.

2. The $\mathscr{P}$-kernel of a semigroup. We now turn our attention to a different kind of kernel of a semigroup. Let $\mathscr{P}$ denote the set of all prime ideals of $S$. We now characterize the $\mathscr{P}$-kernel of $S$. First we need some notation and definitions.

To say that $S$ is a band means that $\mathbf{S}$ is an idempotent semigroup. $S$ is said to be a rectangular band if and only if $S$ is a band and $a b a=a$ for all $a$ and $b$ in $S$. Rectangular bands may be characterized as semigroups of the form $X \times Y$ where $X$ and $Y$ are arbitrary sets and where the operation on $X \times Y$ is defined by

$$
(x, y)\left(x^{\prime}, y^{\prime}\right)=\left(x, y^{\prime}\right)
$$

for $x, x^{\prime}$ in $X$ and $y, y^{\prime}$ in $Y$ (see, for example, [4] or [7]).

We assume, from this point on, that $S$ is any semigroup, that $E$ is the maximal semilattice homomorphic image of $S$, and that $\eta$ is 
the natual homomorphism from $S$ onto $E$. Define a relation $\varphi$ on $S$ by $(a, b) \in \varnothing$ if and only if there exists $x \in S^{1}, y \in S^{1}, c \in S, d \in S$, and positive integers $m$ and $n$ such that $a=x c y, b=x d y$, and $c^{m}=d^{n}$. Clifford has observed, [1], that if $\varphi^{t}$ is the transitive closure of $\varphi$, then $S / \phi^{t}$ is the maximal band homomorphic image of $S$. He also noted that the maximal semilattice homomorphic image of $S / \varphi^{t}$ is the maximal semilattice homomorphic image of $S$. Each $\varphi^{t}$-congruence class of $S$ will be called an archimedean component of $S$. This definition, which agrees with the usual one in case $S$ is commutative, has not been used before in case $S$ is not commutative. Clifford's observation may be rephrased, "any semigroup is a semilattice union of semigroups each of which is a rectangular band of archimedean components of $S$ '.

The following theorem is due to Petrich (see [8] and [9]).

THEorem 2.1. (Petrich) In order that $P$ be a prime ideal of the semigroup $S$ it is necessary and sufficient that there exists a prime ideal $Q$ of $E$ such that $P=\bigcup_{e \in Q} \eta^{-1}(e)$.

The following corollary is immediate.

CoRollary 2.2. The $\mathscr{P}$-kernel of the semigroup $S$ is precisely the inverse image of the $\mathscr{P}^{-k e r n e l}$ of $E$ under $\eta$ (even in case either is void).

Lemma 2.3. If $E$ is a semilattice, then the $\mathscr{P}$-kernel of $E$ is void in case $E$ contains no zero element and otherwise is the zero of $E$.

Proof. Suppose $z$ is in the $\mathscr{P}$-kernel of $E$. If $z$ were not a zero of $E$, then $\{x \in E \mid x \nsupseteq z\}$ would be a prime ideal of $E$ which does not contain $z$.

The next theorem follows immediately from previous results.

THEOREM 2.4. The $\mathscr{S}$-kernel of the semigroup $S$ is void in case $E$ does not contain a zero and otherwise is the inverse image of the zero of $E$ under $\eta$.

CoROLlary 2.5. If the semigroup $S$ contains a zero, then the $\mathscr{P}$-kernel of $S$ is zero if and only if the equations $a c b=0$ and $c^{n}=d^{m}$ imply $a d b=0$ for $a \in S^{1}, b \in S^{1}, c \in S, d \in S$, and positive integers $m$ and $n$. Note that in case $S$ is commutative the latter 
condition merely asserts that 0 is the only nilpotent member of $S$.

Proof. By Theorem 2.4 the $\mathscr{P}$-kernel of $S$ is $\eta^{-1}(z)$ where $z$ is the zero of $E$. Since $\eta^{-1}(z)$ contains the zero of $S$, it must contain only one archimedean component of $S$. Thus the $\mathscr{P}$-kernel of any semigroup with zero is precisely the archimedean component containing the zero. The corollary now follows from the way $\varphi$ was defined.

The following corollaries are evident.

COROLlARY 2.6. The following statements are equivalent:

(1) the maximal semilattice homomorphic image of $S$ is trivial,

(2) the $\mathscr{P}$-kernel of $S$ is $S$, and

(3) $S$ is a rectangular band of its archimedean components.

COROLlary 2.7. The maximal band image of a semigroup is a rectangular band if and only if the maximal semilattice image is trivial.

Finally we consider an application to semilattice theory. To say that $F$ is a face of a semilattice $E$ means that $F$ is a (nonvoid) subsemigroup of $E$ such that either $F$ is $E$ or the complement of $F$ in $E$ is a prime ideal of $E$. A prime ideal $P$ of $E$ is principal if and only if it is of the form $\{x \in E \mid e \geqq x\}$ for some $e \in E$ (in this case $e$ is called the generator of $P$ ).

THEOREM 2.7. If $E$ is a semilattice, then each proper face of $E$ is finite if and only if

(1) each proper prime ideal of $E$ is principal,

(2) each ascending chain in $E$ is finite, and

(3) each nonzero element of $E$ is covered by at most a finite number of elements of $E$.

Proof. First assume each proper face of $E$ is finite. If $P$ is a proper prime ideal of $E$, then $P$ is principal and has as generator the product of all elements of $E$ not in $P$. It is equally clear that (2) and (3) follow.

Now assume (1), (2), and (3) are true. Let $F$ denote any proper face of $E$. Then $E \backslash F$ is a proper prime ideal and thus is principal. Let $e$ denote the generator of $E \backslash F$. Then $x \in F$ if and only if $x \geqq e$, Define a sequence $A$ of subsets of $E$ inductively by

(i) $x \in A_{1}$ if and only if $x \in E$ and $x$ covers $e$, and (ii) if $k$ is a positive integer, $x \in A_{k+1}$ if and only if $x \in E$ and $x$ covers some member of $A_{k}$. 
For each positive integer $i, A_{i}$ is finite. One can show that there is a positive integer $n$ such that $A_{n}$ is void by assuming otherwise and by constructing an infinite ascending chain in $E$. Thus

$$
F=\bigcup_{i=1}^{n} A_{i}
$$

and $F$ is finite. The theorem follows.

If $\mathscr{J}$ is any collection of ideals of a semigroup $S, S /(\cap \mathscr{J})$ is always a subdirect sum of the collection $\{S / T \mid T \in \mathscr{J}\}$. In case $S=E$ is a semilattice the intersection of the collection of all prime ideals of $E$ is void or is a zero of $E$. Thus one obtains the following corollary of Theorem 2.7.

Corollary 2.8. Assume $E$ is a semilattice such that

(1) each proper prime ideal of $E$ is principal,

(2) each ascending chain in $E$ is finite, and

(3) each nonzero element of $E$ is covered by at most a finite number of elements of $E$.

Then $E$ is a subdirect sum of the collection $\left\{F^{\circ} \mid F\right.$ is a finite face of $E\}$.

3. Relationships among various kernels. As in the previous section $S$ denotes any semigroup, $E$ its maximal semilattice homomorphic image, and $\eta$ the natural homomorphism from $S$ onto $E$. Throughout this section $K_{T}$ will denote the intersection of all ideals of the semigroup $T$ and will be called the kernel of $T$. If $N$ denotes the void set, we define $K_{N}=N$. Likewise $P_{T}$ and $M_{T}$ will denote the $\mathscr{P}$ and $\mathscr{C}$ kernels of $T$ respectively.

Theorem 3.1. If $A$ is an ideal of the semigroup $S$, then $K_{A}=K_{S}$. Thus we have

$$
K_{S}=K_{P_{S}}=K_{M_{S}}
$$

Proof. Let $A$ denote any ideal of $S$. If $K_{S}$ is not void, then for each ideal $J$ of $A$

$$
K_{S}=K_{S} J K_{S} K_{S} \subseteq K_{S} J K_{S} \cdot
$$

Thus $K_{S} J K_{S}$ is an ideal of $K_{S}$. Since $K_{S}$ is simple,

$$
K_{S}=K_{S} J K_{S} \subseteq A J A \subseteq J .
$$

Thus $K_{S} \subseteq K_{A}$.

Conversely, if $K_{A}$ is not void, then $K_{S}$ is equal to the intersection 
of the collection $\mathscr{H}$ where $J \in \mathscr{C}$ if and only if $J=I \cap A$ for some ideal $J$ of $S$. But each such $J$ is an ideal of $A$, thus $K_{S} \subseteq K_{A}$.

It follows that $K_{S}=K_{A}$ for each ideal $A$ of $S$. Clearly if $P_{S}$ or $M_{S}$ is void so is $K_{S}$. The theorem follows.

CoRollary 3.2. If $S$ is a semigroup, then the kernel of $S$ is the same as the kernel of $P_{S}$ and thus is the kernel of a rectangular band of archimedean components of $S$.

In order to obtain the relationship between the $\mathscr{C l}$-kernel, $M_{S}$, and the $\mathscr{P}$-kernel, $P_{S}$, we need more information about the maximal ideals of $S$. The next theorem provides such information and has some interest in its own right. First we need another definition. An ideal $I$ of $\eta^{-1}(e)$, for $e \in E$, is said to be induced by $S$ if and only if $I \cup\left(S \backslash \eta^{-1}(e)\right)$ is an ideal of $S$. It is easy to see that an ideal $I$ of $\eta^{-1}(e)$ is induced by $S$ if and only if

(1) $f_{1} \in E \backslash\{e\}, f_{2} \in E \backslash\{e\}$, and $f_{1} f_{2}=e$ imply $\eta^{-1}\left(f_{1}\right) \eta^{-1}\left(f_{2}\right) \subseteq I$ and

(2) $f \in E$ and $f>e$ imply $\eta^{-1}(f) I \subseteq I$ and $I \eta^{-1}(f) \subseteq I$.

THEOREM 3.3. If $M$ is a subset of the semigroup $S$, then $M$ is a maximal ideal of $S$ if and only if there exists $e \in E$ such that either

(1) $e$ is a maximal element of $E$ such that $\eta^{-1}(e)$ is simple and $M=\mathbf{U}_{f \epsilon_{E \backslash[e]}} \eta^{-1}(f)$, or

(1) there is a maximal ideal $M_{e}$ of $\eta^{-1}(e)$ such that $M_{e}$ is induced by $S$ and $M=M_{e} \cup \bigcup_{f \in E \backslash\{e\}} \eta^{-1}(f)$.

Proof. Suppose $M$ is a maximal ideal of $S$ and that $a \in S \backslash M$. Let $e$ denote $\eta(a)$. First we show that $\eta^{-1}(f) \cong M$ for all $f \in E \backslash\{e\}$. Consider $F=\{x \in E \mid x \supsetneqq e\}$. The set $M \cup \eta^{-1}(F)$ is a proper ideal of $S$. Since $M$ is maximal, $\eta^{-1}(F) \subseteq M$. Thus if $f \in E$ such that $\eta^{-1}(f) \nsubseteq M$, then $e \leqq f$. Suppose there exists $f_{0} \in E$ such that

$$
\eta^{-1}\left(f_{0}\right) \nsubseteq M
$$

such that $f_{0}>e$. If $F_{0}=\left\{x \in E \mid x \nsupseteq f_{0}\right\}$, then $M \cup \eta^{-1}\left(F_{0}\right)$ is a proper ideal of $S$. Thus $\eta^{-1}\left(F_{0}\right) \subseteq M$. But $f_{0}>e$ implies that $e \in F_{0}$ and that $\eta^{-1}(e) \supseteqq M$, contrary to the choice of $e \in E$. It follows that $\eta^{-1}(f) \subseteq M$ for each $f \neq e$.

We now show that if $M \cap \eta^{-1}(e)$ is not void, then $M_{e}=M \cap \eta^{-1}(e)$ is a maximal ideal of $\eta^{-1}(e)$ which is induced by $S$. Suppose there is an ideal $J$ of $\eta^{-1}(e)$ such that $M_{e} \subset J \subset \eta^{-1}(e)$. Then $M \cup J$ is an ideal of $S$ such that $M \subset M \cup J \subset S$. Thus no such $J$ exists and $M_{0}$ is a maximal ideal of $\eta^{-1}(e)$. Clearly $M_{e}$ is induced by $S$. 
Similar reasoning shows that if $M \cap \eta^{-1}(e)$ is void, then $e$ is maximal in $E$ and that $\eta^{-1}(e)$ is simple.

The proof of the other half of the theorem is easy and is omitted.

Lemma 3.4. The $\mathscr{C}$-kernel of a semigroup is never void.

Proof. Assume the $\mathscr{C}$-kernel of some semigroup $S$ is void. Then the $/ C$-kernel of $S^{\circ}$ is zero and thus $S^{\circ}$ is a mutually annihilating sum of semigroups each of which either is a null semigroup of order two or is a simple semigroup with zero. Since 0 is a prime ideal of $S^{\circ}, S^{\circ}$ must be a simple semigroup with zero. Thus $S$ is simple and the $\mathscr{C}$-kernel of $S$ is $S$ contrary to the assumption that the $/ t$-kernel of $S$ is void.

THEOREM 3.5. In order that the $/$-kernel of $S$ be a subset of the $\mathscr{S}$-kernel of $S$ it is necessary and sufficient that $E$ contain a zero $z$ and that for cach $e \in E \backslash\{z\}, e$ is maximal in $E$ and $\eta^{-1}(e)$ is simple.

REMARK. We do not require in the previous theorem that $E$ contain elements other than $z$.

Proof. Assume $M_{S} \subseteq P_{S}$. Since $M_{S}$ is not void, neither is $P_{S}$; thus there is a zero $z$ in $E$ and $P_{S}=\eta^{-1}(z)$. Assume $e \in E \backslash\{z\}$. We show that $e$ is maximal in $E$ and that $\eta^{-1}(e)$ is simple. To do this it suffices, by Theorem 3.3, to show that $\mathbf{U}_{f \in E \backslash\{e\}} \eta^{-1}(f)$ is a maximal ideal of $S$. Assume $\bigcup_{f \in E \backslash\{e\}} \eta^{-1}(f)$ is not a maximal ideal of $S$. Since $M_{S} \subseteq P_{S}, x \in \eta^{-1}(e)$ implies that there exists a maximal ideal $M_{x}$ of $S$ such that $x \notin M_{x}$. Since $\bigcup_{f \in E \backslash\{e\}} \eta^{-1}(f)$ is not a maximal ideal of $S$, Theorem 3.3 implies that there exists a maximal ideal $N_{x}$ of $\eta^{-1}(e)$ such that $M_{x}=N_{x} \cup \bigcup_{f \in E \backslash(e)} \eta^{-1}(f)$. Since for each $x \in \eta^{-1}(e), x \notin N_{x}$, we have that $\bigcap_{x \in \eta^{-1}(e)} N_{x}$ is void. But $\bigcap_{x \in \eta^{-1}(e)} N_{x}$ contains the $\mathscr{C}$ kernel of $\eta^{-1}(e)$ which, by the lemma, is not void. We have established the necessity of the condition.

Now assume $E$ has a zero and that if $e \in E \backslash\{z\}$, then $e$ is maximal in $E$ and $\eta^{-1}(e)$ is simple. If $E$ contains no element other than $z$, then $M_{S} \subseteq S=\eta^{-1}(z)=P_{S}$. Assume $E$ contains elements other than z. For each $e \in E \backslash\{z\}$ it is easy to see that $\bigcup_{f E \backslash\{e\}} \eta^{-1}(f)$ is a maximal ideal of $S$. Thus $\bigcap_{e \in E \backslash\{z\}}\left[\mathrm{U}_{f \in E \backslash \ell e\}} \eta^{-1}(f)\right]=\eta^{-1}(z)$ contains $\bigcap \mathscr{l}$ and $M_{S} \subseteq \eta^{-1}(z)=P_{S}$. The theorem follows.

CoRollary 3.6. If $E$ has a zero $z$ and $\eta^{-1}(z)$ is simple, then. the following statements are equivalent:

(1) $M_{S}=P_{S}$, and

(2) whenever $e \in E \backslash\{z\}$, $e$ is maximal in $E$ and $\eta^{-1}(e)$ is simple. 


\section{REFERENCES}

1. A. H. Clifford, Review of [14], Math. Reviews 17 (1956), 584.

2. A. H. Clifford and G. B. Preston, The algebraic theory of semigroups, Math. Surveys No. 7, Amer. Math. Soc., Providence, R. I., 1961.

3. H.-J. Hoehnke, Über des untere und obere Radical einer Halbgruppe, Math. Zeitschr. 89 (1965), 300-311.

4. N. Kimura, The structure of idempotent semigroups I., Pacific J. Math. 8 (1958), 257-275.

5. G. Lallement and M. Petrich, Some results concerning completely 0-simple semigroups, Bull. Amer. Math. Soc. 70 (1964), 777-778.

6. E. S. Ljapin, Normal complexes of associative systems, Izn, Acad. Nauk SSSR Ser. Mat. 14 (1950), 179-192. (Russian)

7. D. McLean, Idempotent semigroups, Amer. Math. Monthly 61 (1954), 110-113.

8. M. Petrich, The maximal semilattice decomposition of a semigroup, Bull. Amer. Math. Soc. 69 (1963), 342-344.

9. - - The maximal semilattice decomposition of a semigroup, Math. Zeitschr. 85 (1964), 68-82.

10. S. Schwarz, On dual semigroups, Czech. Math. J. 10 (1960), 201-230.

11. - On maximal ideals in the theory of semigroups I, Czech. Math. J. 3 (1953), 139-153.

12. On maximal ideals in the theory of semigroups II, Czech. Math. J. 3 (1953), 365-383.

13. H. Seidel, Über das Radikal einer Halbgruppe, Math. Nachr. 29 (1965), 255-263.

14. M. Yamada, On the greutest semilattice decomposition of a semigroup, Ködai Math. Sem. Rep. 7 (1955), 59-62.

Received June 15, 1966 and in revised form May 5, 1967. The author wishes to acknowledge support by NASA, Grant NGR-44-005-037.

UNIVERSITY OF HOUSTON 



\section{PACIFIC JOURNAL OF MATHEMATICS}

\section{EDITORS}

\section{H. ROYDEN}

Stanford University

Stanford, California

\section{J. P. Jans}

University of Washington

Seattle, Washington 98105

\section{J. DUGUNDJI}

Department of Mathematics

Rice University

Houston, Texas 77001

RICHARD ARENS

University of California

Los Angeles, California 90024

\section{ASSOCIATE EDITORS}
E. F. BeCKenbaCH
B. H. NeumanN
F. WOLF
K. YosIDA

\section{SUPPORTING INSTITUTIONS}

UNIVERSITY OF BRITISH COLUMBIA CALIFORNIA INSTITUTE OF TECHNOLOGY UNIVERSITY OF CALIFORNIA MONTANA STATE UNIVERSITY UNIVERSITY OF NEVADA NEW MEXICO STATE UNIVERSITY OREGON STATE UNIVERSITY UNIVERSITY OF OREGON OSAKA UNIVERSITY UNIVERSITY OF SOUTHERN CALIFORNIA
STANFORD UNIVERSITY UNIVERSITY OF TOKYO UNIVERSITY OF UTAH WASHINGTON STATE UNIVERSITY UNIVERSITY OF WASHINGTON

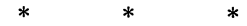

AMERICAN MATHEMATICAL SOCIETY CHEVRON RESEARCH CORPORATION TRW SYSTEMS

NAVAL ORDNANCE TEST STATION 


\section{Pacific Journal of Mathematics}

\section{Vol. 24, No. $1 \quad$ May, 1968}

Harry P. Allen, Lie algebras of type $D_{4}$ over algebraic number fields ...... 1

Charles Ballantine, Products of positive definite matrices. II............ 7

David W. Boyd, The spectral radius of averaging operators ............ 19

William Howard Caldwell, Hypercyclic rings ................... 29

Francis William Carroll, Some properties of sequences, with an application

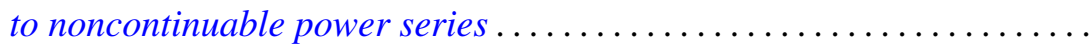

David Fleming Dawson, Matrix summability over certain classes of

sequences ordered with respect to rate of convergence ........... 51

D. W. Dubois, Second note on David Harrison's theory of preprimes. . . . . 57

Edgar Earle Enochs, A note on quasi-Frobenius rings.............. 69

Ronald J. Ensey, Isomorphism invariants for Abelian groups modulo bounded groups ................................ 71

Ronald Owen Fulp, Generalized semigroup kernels ................ 93

Bernard Robert Kripke and Richard Bruce Holmes, Interposition and approximation ................................. 103

Jack W. Macki and James Sai-Wing Wong, Oscillation of solutions to second-order nonlinear differential equations ..................

Lothrop Mittenthal, Operator valued analytic functions and generalizations

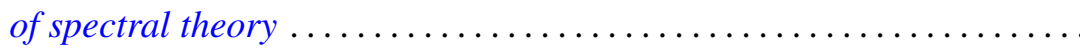

T. S. Motzkin and J. L. Walsh, A persistent local maximum of the pth power deviation on an interval, $p<1 \ldots \ldots \ldots \ldots \ldots \ldots \ldots \ldots \ldots \ldots . \ldots \ldots$

Jerome L. Paul, Sequences of homeomorphisms which converge to homeomorphisms ...........................

Maxwell Alexander Rosenlicht, Liouville's theorem on functions with elementary integrals.

Joseph Goeffrey Rosenstein, Initial segments of degrees .

$\mathrm{H}$. Subramanian, Ideal neighbourhoods in a ring ............

Dalton Tarwater, Galois cohomology of abelian groups . .

James Patrick Williams, Schwarz norms for operators ... .

Raymond Y. T. Wong, A wild Cantor set in the Hilbert cube. 